\title{
Comparison of Videofluoroscopic Swallowing Study and Radionuclide Salivagram for Aspiration Pneumonia in Children With Swallowing Difficulty
}

\author{
Go Eun Kim, $\mathrm{MD}^{1}$, In Young Sung, MD, $\mathrm{PhD}^{1}$, Eun Jae Ko, $\mathrm{MD}^{1}$, \\ Kyoung Hyo Choi, $\mathrm{MD}, \mathrm{PhD}^{1}$, Jae Seung Kim, $\mathrm{MD}, \mathrm{PhD}^{2}$ \\ Departments of ${ }^{1}$ Rehabilitation Medicine and ${ }^{2}$ Nuclear Medicine, Asan Medical Center, \\ Ulsan University College of Medicine, Seoul, Korea
}

\begin{abstract}
Objective To determine whether the use of both videofluoroscopic swallowing study (VFSS) and radionuclide salivagram was beneficial for detecting aspiration-induced pneumonia in children with swallowing difficulty.

Methods From 2001 to 2016, children who underwent both VFSS and salivagram consecutively for suspected aspiration or dysphagia were included in the study. Demographic data, findings of VFSS and salivagram, and medical records were reviewed.

Results Aspiration pneumonia (AP) was present in 34 out of 110 children; 48 showed positive aspiration findings in VFSS and 33 showed positive aspiration findings in salivagram. Among the 62 children who were negative of aspiration in VFSS, $12(19.4 \%)$ showed positive aspiration findings in salivagram. Four out of 12 children were diagnosed with AP. The aspiration findings in both VFSS and salivagram were significantly related to AP. However, the aspiration findings in the two tests were weakly consistent. Even if one test showed negative aspiration, it was helpful to additionally detect AP using another test, which showed positive aspiration finding. If aspiration findings were positive in only one of the two tests, the probability of AP was $38.5 \%$, whereas if they were positive in both tests, the probability increased to $66.7 \%$. If the aspiration findings were negative in both tests, AP did not occur with a probability of $90 \%$.

Conclusion Salivagram is a valuable tool for monitoring of aspiration in children with swallowing difficulties. It could be helpful in assessment of children at a high risk of AP, even if the VFSS showed negative aspiration findings. Thus, testing for AP using both VFSS and salivagram is desirable.
\end{abstract}

Keywords Child, Swallowing disorders, Pneumonia, Aspirations, Salivagram, Videofluoroscopic swallowing study

Received June 14, 2017; Accepted July 3, 2017

Corresponding author: In Young Sung

Department of Rehabilitation Medicine, Asan Medical Center, University of Ulsan College of Medicine, 88 Olympic-ro 43-gil, Songpa-gu, Seoul 05505, Korea. Tel: +82-2-3010-3800, Fax: +82-2-3010-6964, E-mail: iysung56@gmail.com

ORCID: Go Eun Kim (http://orcid.org/0000-0003-1481-1317); In Young Sung (http://orcid.org/0000-0001-6545-6744); Eun Jae Ko (http://orcid. org/0000-0001-7198-5407); Kyoung Hyo Choi (http://orcid.org/0000-0001-9137-3889); Jae Seung Kim (http://orcid.org/0000-0003-1710-1185).

(c) This is an open-access article distributed under the terms of the Creative Commons Attribution Non-Commercial License (http://creativecommons.org/ licenses/by-nc/4.0) which permits unrestricted noncommercial use, distribution, and reproduction in any medium, provided the original work is properly cited. Copyright $\odot 2018$ by Korean Academy of Rehabilitation Medicine 


\section{INTRODUCTION}

In children, swallowing difficulties may be attributed to congenital abnormalities involving swallowing-related organs, developmental delay, and abnormalities in the central nervous system. Swallowing difficulty is frequently encountered, especially in children with underlying diseases. According to previous studies, swallowing disorders have been reported in $43 \%-100 \%$ of children with cerebral palsy $[1,2]$ and $35 \%-80 \%$ of children with neuromuscular disease [3]. Swallowing difficulty may lead to life-threatening conditions, such as aspiration pneumonia (AP), asphyxia, and sepsis. Therefore, it is important to evaluate and diagnose swallowing difficulty in pediatric patients. However, physicians should consider that the anatomic structures of children differ from those of adults and that evaluation of swallowing in children could be limited.

Among the several subjective and objective methods available, videofluoroscopic swallowing study (VFSS) and salivagram are mainly used in children. VFSS, considered as the gold standard for examining the oral and pharyngeal mechanisms of dysphagia in adults, offers detailed information regarding the anatomy and physiology of swallowing. It is widely practiced in both adults and children, however, this method may be limited or difficult to perform in infants or children who show poor cooperation.

Salivagram is a scintigraphic method that enables detection of salivary aspiration into the lungs. Salivagram can be easily and safely conducted with relatively low radiation exposure [4]. Salivagram has a high detection rate for pulmonary aspiration [5] and is known to detect salivary aspiration more sensitively than laryngoscopy in brain-injured patients with tracheostomy [6]. Positive signs in salivagrams are related to respiratory infection and/or pneumonia in infants [7]. Especially in children, the advantage of salivagram is that it can be used to evaluate children in whom there is difficulty in performing VFSS.

In clinical practice, cases requiring both VFSS and salivagram are frequently encountered. In most cases, the selection of the tests is determined by the physician's experience. Baikie et al. [8] reported a 59.3\% (kappa val$\mathrm{ue}=0.20$ ) agreement between VFSS and salivagram findings. In a study of elderly population [9], the combination of VFSS and salivagram led to a more effective diagnosis of AP. However, the need for either one or both VFSS and salivagram in children remains to be elucidated. We hypothesized that the combination of both VFSS and salivagram in children with swallowing difficulty is clinically advantageous compared with a single option. Accordingly, we investigated the effectiveness of both VFSS and salivagram in predicting AP in children with swallowing difficulty.

\section{MATERIALS AND METHODS}

\section{Subjects}

We retrospectively reviewed pediatric patients with swallowing difficulty who underwent both VFSS and salivagram from January 2001 to June 2016. Both VFSS and salivagram were consecutively used to evaluate patients with suspected aspiration or swallowing difficulty. An interval of $<7$ days was regarded as consecutive (the mean difference in time between two test days was 2.91 days). Baseline characteristics, such as age, sex, underlying disease, feeding method, body mass index, and presence of tracheostomy, were investigated.

The underlying diseases were largely divided into neurological and non-neurological diseases [10]. The feeding methods were divided into oral and non-oral diets. Oral diet was further divided into regular and limited diets. Non-oral diet indicated feeding via nasogastric, orogastric, gastric, or jejunostomy tubes.

\section{Aspiration pneumonia}

A retrospective chart review was conducted to confirm the occurrence of AP in the subjects. The following data were collected: symptoms such as coughing or choking during or after feeding; sputum presence, dyspnea, or fever; chest X-ray findings; laboratory findings (white blood cell counts and C-reactive protein levels); blood culture results, and antibiotic use.

Although a definitive diagnosis of AP is difficult and the diagnostic criteria for AP differ slightly across studies [2,11-13], patients who met all the following criteria were considered to have AP in this study: (1) the presence of both subjective symptoms (fever, cough, and sputum production) and objective signs suggesting pneumonia (coarse lung sounds, presence of lung infiltration on chest X-ray, and systemic inflammation, based on labo- 
ratory findings such as white blood cell counts and C-reactive protein levels), (2) clinical suspicion of aspiration (choking or delayed swallowing), and (3) no evidence of microorganisms, such as legionella or mycoplasma, which are responsible for atypical pneumonia [12,13]. In addition, the clinical reports of pediatricians were used for reference purposes.

\section{VFSS measurement}

VFSS was performed with the patient in an upright seated position, and when it was difficult, the caregiver assisted the patient in maintaining an upright posture. VFSS was performed by an experienced physician, who was assisted by a radiologic technician in the fluoroscopic laboratory. Data were recorded on a video recorder Sonialvision G4 (Shimadzu, Tokyo, Japan). If the patient was an infant receiving a liquid diet, a feeding bottle filled with a thin liquid was provided to observe the sucking power and aspiration or penetration into the airway during swallowing. In children who chewed and swallowed regular diets, protocols similar to those used for adults were used; these children were administered a thick barium fluid mixture, pureed diet, mechanically-altered diet, and thin barium fluid to swallow twice. When the food material entered the airway below or above the vocal folds, the condition was referred to as subglottic aspiration or supraglottic penetration, respectively. Furthermore, the presence of residual materials in the vallecular pouch or pyriform sinus, abnormal findings in the oral and esophageal phases, and scores on the American Speech-Language-Hearing Association National Outcomes Measurement System (ASHA-NOMS) and Penetration-Aspiration Scale (PAS) were recorded. ASHANOMS consists of a 7-point scale according to the level of the patient's dietary restriction. Level 1 refers to the stage in which the patient cannot eat per os and requires a non-oral diet, and level 7 indicates that the patient can swallow without restriction. PAS is an 8-point evaluation scale evaluating penetration and aspiration events during VFSS. If the food material does not enter the airway, it is designated as grade 1 , and the grade increases as the material enters the airway deeper below the vocal cord. In this study, subglottic aspiration of PAS score $\geq 6$ was defined as 'aspiration' in VFSS.

\section{Radionuclide salivagram measurement}

Salivagram is a test used to detect salivary aspiration by swallowing radionuclide material and assessing whether radioactivity remains in the airway. Salivagram was performed with diluted Tc-99m diethylenetriamine pentaacetic acid (DTPA). A small drop of diluted Tc-99m DTPA (3.7 MBq) was administered under the tongue of each patient in a supine position on the imaging table. The patient was induced to swallow saliva naturally, and images of the mouth, chest, and upper abdomen were then obtained. The dynamic acquisition of anterior and posterior images for the first $10 \mathrm{~min}$, followed by delayed static images at 10 and 120 minutes, was performed. Images were analyzed by experienced nuclear medicine physicians. Aspiration was defined as detectable radiopharmaceutical activity within the tracheobronchial fields $[4,9]$.

\section{Statistical analysis}

Statistical analyses were performed using the SPSS version 18.0 for Windows (SPSS Inc., Chicago, IL, USA). All data were expressed as mean \pm standard deviation. Statistical significance was defined as a $p$-value of $<0.05$. For the comparison between groups, the independent $t$-test was used if variances were equal; otherwise, the MannWhitney U-test was used for analysis. The odds ratios were determined using the chi-square test to evaluate the association of AP with VFSS and salivagram. Linearby-linear association analysis was used to determine the linear correlation between AP and abnormal findings in VFSS and salivagram. Kappa value analysis was used to assess the degree of agreement between VFSS and salivagram findings.

\section{RESULTS}

\section{Patient characteristics}

During 2006-2016, 117 pediatric patients underwent VFSS and salivagram consecutively for the evaluation of swallowing or feeding difficulty at a single center. A total of 110 patients were included. There were 65 males and 45 females with an age range of 1-192 months (mean, 28.3 months). Among the 110 patients, three (2.7\%) had no underlying disease. Among neurological diseases, hypoxic brain injury and cerebral palsy were the most common, and among non-neurological diseases, cardiopulmonary disease was the most common (Table 1). 
In terms of current feeding, 44 patients $(40.0 \%)$ were on oral feeding and $58(52.7 \%)$ received non-oral feeding. Seventeen patients (15.5\%) underwent tracheostomy.

In this study, all the enrolled patients underwent both VFSS and salivagram, and the mean difference in time between the two tests was 2.91 days (median, 2 days; interquartile range, $1-5$ days).

Based on the clinical, radiological, and laboratory criteria, AP was diagnosed in $34(30.9 \%)$ of 110 patients. Among them, 26 patients $(76.4 \%)$ had fever (body temperature, $\left.\geq 37.8^{\circ} \mathrm{C}\right), 12(35.3 \%)$ reported coughing during feeding, and three (8.8\%) reported vomiting. Among 32 patients undergoing blood culture, no bacterial growth was found in 31 while Staphylococcus aureus was detected in one patient. Thirty-two of the 34 patients diagnosed with AP (94.1\%) were prescribed antibiotics, and the mean duration of medication was 10.7 days.

\section{Aspiration findings in VFSS or salivagram and} correlation between the two tests

In total, 48 children (43.6\%) showed positive aspiration findings in VFSS and 33 (30.3\%) showed positive aspira-

Table 1. Baseline characteristics $(\mathrm{n}=110)$

\begin{tabular}{|cc}
\hline \multicolumn{1}{c}{ Characteristic } & Value \\
\hline Age $(\mathrm{mo})$ & $28.32 \pm 38.98$ \\
\hline Gender $($ male:female) & $65: 45$ \\
\hline BMI $\left(\mathrm{kg} / \mathrm{m}^{2}\right)$ & $15.30 \pm 2.51$ \\
\hline Time difference between two studies (day) & $2.91 \pm 2.53$ \\
\hline Underlying disease & \\
\hline None & $3(2.7)$ \\
\hline Neurological & $52(47.3)$ \\
\hline Non-neurological & $55(50.0)$ \\
The method of current feeding & \\
\hline Oral feeding & $44(40.0)$ \\
\hline Regular diet & $34(30.6)$ \\
\hline Limited diet & $10(9.0)$ \\
\hline Non-oral feeding & $58(52.7)$ \\
\hline Nasogastric or orogastric tube & $47(42.3)$ \\
\hline Gastric or jejunostomy tube & $11(9.9)$ \\
\hline Oral+non-oral feeding & $8(7.2)$ \\
\hline Presence of aspiration pneumonia & $34(30.9)$ \\
\hline Presence of tracheostomy & $17(15.5)$ \\
\hline
\end{tabular}

Values are presented as mean \pm standard deviation or number (\%).

BMI, body mass index. tion findings in salivagram. Twenty-one children showed positive aspiration findings in both tests, and 50 children showed negative aspiration findings in both tests. The remaining 39 children showed positive aspiration findings in only one of the two tests (Table 2). Thus, the probability of the same result (normal or abnormal) in both tests known as 'total agreement' was $64.5 \%(71 / 110)$. When the consistency between the two tests was calculated using kappa value analysis, the value was $0.21(\mathrm{p}=0.03)$, indicating weak consistency.

\section{Correlation of the two test results with AP}

AP was significantly correlated with ASHA-NOMS scores $(\mathrm{p}<0.001)$, positive aspiration findings in VFSS $(\mathrm{p}<0.001)$, and positive aspiration findings in salivagram $(p<0.001)$. Abnormal findings in the oral phase of VFSS and the presence of gastroesophageal reflux were not significantly different between the groups with and without AP. In the presence of aspiration findings on VFSS or salivagram, the odds ratios for AP were $4.50(\mathrm{p}<0.001)$ and $4.58(\mathrm{p}<0.001)$, respectively.

\section{Relationship between AP and findings in VFSS and/or salivagram}

Among 48 children with positive aspiration findings in VFSS, 25 (52.1\%) had AP. Meanwhile, among 62 children with negative aspiration finding in VFSS (Table 3), nine were diagnosed with AP (14.5\%). Although in the group with negative aspiration finding in VFSS, 12 children (19.4\%) showed positive aspiration findings in salivagram and 4 of $12(33.3 \%)$ had AP.

In salivagram, positive aspiration findings were observed in 33 children, and 18 (54.5\%) were diagnosed with AP. Among the 77 children with negative aspiration findings in salivagram (Table 4), AP was detected in 16

Table 2. Correlation of AP with VFSS and salivagram results

\begin{tabular}{lccc}
\hline & \multicolumn{3}{c}{ Salivagram } \\
\cline { 2 - 4 } & Aspiration (+) & Aspiration (-) & Total \\
\hline VFSS & & & \\
Aspiration (+) & 21 & 27 & 48 \\
Aspiration (-) & 12 & 50 & 62 \\
Total & 33 & 77 & 110 \\
\hline
\end{tabular}

$\mathrm{AP}$, aspiration pneumonia; VFSS, videofluoroscopic swallowing study. 
Table 3. Correlation between salivagram findings and AP in the normal VFSS group $(\mathrm{n}=62)$

\begin{tabular}{lccc}
\hline & \multicolumn{3}{c}{ Salivagram } \\
\cline { 2 - 4 } & Aspiration (+) & Aspiration (-) & Total \\
\hline AP (+) & $4(33.3)$ & $5(10.0)$ & $9(14.5)$ \\
AP (-) & $8(66.6)$ & $45(90.0)$ & $53(85.5)$ \\
Total & $12(19.4)$ & $50(80.6)$ & $62(100)$ \\
\hline
\end{tabular}

Values are expressed as number (\%).

AP, aspiration pneumonia; VFSS, videofluoroscopic swallowing study.

p-value $(=0.06)$ and odds ratio $(=3.57)$ were calculated using the chi-square test.

Table 4. Correlation between VFSS findings and AP in the normal salivagram group $(\mathrm{n}=77)$

\begin{tabular}{lccc}
\hline & \multicolumn{3}{c}{ VFSS } \\
\cline { 2 - 4 } & Aspiration (+) & Aspiration (-) & Total \\
\hline AP (+) & $11(40.7)$ & $5(10.0)$ & $16(20.8)$ \\
AP (-) & $16(59.3)$ & $45(90.0)$ & $61(79.2)$ \\
Total & $27(35.1)$ & $50(64.9)$ & $77(100)$ \\
\hline
\end{tabular}

Values are expressed as number (\%).

VFSS, videofluoroscopic swallowing study; AP, aspiration pneumonia.

p-value $(=0.01)$ and odds ratio $(=4.33)$ were calculated using the chi-square test.

children. In this group with negative aspiration findings in salivagram, 27 (35.1\%) showed positive aspiration findings in VFSS and 11 of them (40.7\%) had AP.

When the aspiration findings were positive in only one of the two tests, the probability of AP was $38.5 \%$, but if aspiration findings were positive in both tests, the probability increased to $66.7 \%$ with statistical significance. If aspiration findings were negative in both tests, AP did not occur with a probability of $90 \%$ (Table 5 ).

\section{DISCUSSION}

Swallowing difficulty in children is associated with morbidity and mortality. VFSS and radionuclide salivagram are commonly used in clinical settings to assess swallowing difficulties in children.

VFSS is the gold standard for assessment of oral and pharyngeal abnormalities associated with swallowing in real time. VFSS can sensitively detect pulmonary aspiration considering that aspiration mainly occurs 'silently' without coughing in children with cerebral palsy [14].
Table 5. Correlation of salivagram and/or VFSS with AP $(\mathrm{n}=110)$

\begin{tabular}{lrrrr}
\hline & $\begin{array}{c}\text { Aspiration } \\
(+) \text { in two } \\
\text { studies }\end{array}$ & $\begin{array}{c}\text { Aspiration } \\
(+) \text { in one } \\
\text { study }\end{array}$ & $\begin{array}{c}\text { No aspira- } \\
\text { tion in all } \\
\text { studies }\end{array}$ & p-value \\
\hline AP (+) & $14(66.7)$ & $15(38.5)$ & $5(10.0)$ & \\
AP (-) & $7(33.3)$ & $24(61.5)$ & $45(90.0)$ & $<0.001^{*}$ \\
\hline
\end{tabular}

Values are expressed as number (\%).

VFSS, videofluoroscopic swallowing study; AP, aspiration pneumonia.

${ }^{*} \mathrm{p}<0.05$, according to the linear-by-linear association.

However, there are also limitations in performing VFSS in children. In this real-time dynamic study, episodic aspiration may not be accurately reflected during examination, and it is difficult to perform the test if children are very young or have poor cooperation. In addition, there might be restrictions involving the examination time due to radiation exposure [15].

Since its introduction in 1989, salivagram has been used for the diagnosis of saliva aspiration in children and adults to provide additional benefits. Most studies involving salivagram till date have been conducted in children. Abnormal salivagram findings (for e.g., radioactivity in the airway) in children with cerebral palsy are highly suggestive of pulmonary aspiration [16]. Salivagram is an effective tool to confirm salivary aspiration in patients with recurrent pneumonia [17]. In one study conducted in children with cerebral palsy, salivagram was more sensitive in detecting aspiration than VFSS [8]. In addition, the results correlate well with chest X-ray findings [4]. Salivagram can be easily and safely performed, is not relatively limited to infants, and has the advantage of limited radiation exposure.

Although both VFSS and salivagram are frequently used in clinical practice, little research has been conducted on the utility of combining the tests or comparing the test results. One study compared VFSS and salivagram findings in adults with AP [9]. The subjects were generally elderly with a mean age of 68.8 years. Most of them had underlying diseases such as stroke, Parkinson disease, brain tumor, and lung cancer. This study concluded that conducting the tests together can be an effective strategy to investigate AP. Sensitivity to AP was similar in both tests, but VFSS was reported to be slightly more sensitive than salivagram, which is consistent with our findings.

Unlike the study conducted in elderly population, the 
subjects of our study were children, and to the best of our knowledge, this is the first study comparing VFSS and salivagram findings in children with swallowing difficulty. VFSS and salivagram have the aforementioned advantages and disadvantages. However, there is also a limitation in performing the tests, especially VFSS, in children. This study suggests that the tests complement each other, and conducting salivagram together with VFSS provides additional benefit in evaluating children with swallowing difficulty.

Among a total of 110 children, 48 showed positive aspiration findings in VFSS and 33 had positive aspiration finding in salivagram. Twenty-one children had positive aspiration findings in both tests and 50 had negative aspiration finding. Thus, the total agreement of the two tests was $64.5 \%$, which was not significantly elevated and was slightly lower than the values reported in an adult study (72\%) [9]. In addition, the results of both tests showed a weak consistency with each other. Possible explanations for these results are as follows. First, salivagram and VFSS evaluate different types of swallowing: in VFSS, the patient swallows large boluses of food material in the upright posture, whereas in salivagram, patient swallows saliva in the supine position, which occurs unconsciously and the patient swallows comparatively very little amount of food [8]. In addition, episodic aspiration may not be detected by the tests. Finally, the difficulty of performing VFSS in children might interfere with the interpretation of the test and lead to inconsistency between the two tests.

Next, we explored the relationship between the results of the two tests and AP. The presence of positive aspiration findings on VFSS and salivagram was significantly correlated with AP. The consistency between the two tests was not significantly high, and 39 children had incompatible results in both tests. In this case, even if one test showed negative aspiration findings, it was helpful to additionally evaluate AP using another test, which showed positive aspiration findings. For example, in the group with negative aspiration findings on VFSS, nearly $20 \%$ of children showed positive aspiration findings on salivagram, and one-third of the aspirated children had AP.

Similarly, the probability of AP was significantly higher when the aspiration findings were positive in both tests rather than in a single test. When both tests were negative for aspiration, AP did not occur with a probability of
$90 \%$. Taken together, these results suggest that combining salivagram with VFSS may improve the accuracy of AP diagnosis.

Limitation of this study include the retrospective design; hence, data of the presence or absence of AP were obtained only through medical records and we could not investigate if there was any subject loss to follow-up. Second, because only aspiration with PAS score $\geq 6$ on VFSS was defined as 'aspiration' in our study, data of penetration less than PAS score 6 and abnormal findings in oral and esophageal phases were not included in the results. However, considering that salivagram assesses the aspiration of test materials in the airway, we decided to compare the two tests. A previous study [9] also similarly divided VFSS results according to the presence of aspiration.

In conclusion, salivagram is a valuable evaluation tool for children with swallowing difficulty. It enables assessment of children at a high risk of AP, even if VFSS showed negative aspiration findings. Considering the weak consistency between the two tests and specific limitations in pediatric populations, combining VFSS and salivagram increases the accuracy of AP diagnosis.

\section{CONFLICT OF INTEREST}

No potential conflict of interest relevant to this article was reported.

\section{REFERENCES}

1. Erasmus CE, van Hulst K, Rotteveel JJ, Willemsen MA, Jongerius $\mathrm{PH}$. Clinical practice: swallowing problems in cerebral palsy. Eur J Pediatr 2012;171:409-14.

2. Lagos-Guimaraes HN, Teive HA, Celli A, Santos RS, Abdulmassih EM, Hirata GC, et al. Aspiration pneumonia in children with cerebral palsy after videofluoroscopic swallowing study. Int Arch Otorhinolaryngol 2016;20:132-7.

3. Audag N, Goubau C, Toussaint M, Reychler G. Screening and evaluation tools of dysphagia in children with neuromuscular diseases: a systematic review. Dev Med Child Neurol 2017;59:591-6.

4. Drubach LA, Zurakowski D, Palmer EL 3rd, Tracy DA, Lee EY. Utility of salivagram in pulmonary aspiration in pediatric patients: comparison of salivagram and 
chest radiography. AJR Am J Roentgenol 2013;200:43741.

5. Yang J, Codreanu I, Servaes S, Zhuang H. Radionuclide salivagram and gastroesophageal reflux scintigraphy in pediatric patients: targeting different types of pulmonary aspiration. Clin Nucl Med 2015;40:559-63.

6. Kang Y, Chun MH, Lee SJ. Evaluation of salivary aspiration in brain-injured patients with tracheostomy. Ann Rehabil Med 2013;37:96-102.

7. Heyman S, Respondek M. Detection of pulmonary aspiration in children by radionuclide "salivagram". J Nucl Med 1989;30:697-9.

8. Baikie G, South MJ, Reddihough DS, Cook DJ, Cameron DJ, Olinsky A, et al. Agreement of aspiration tests using barium videofluoroscopy, salivagram, and milk scan in children with cerebral palsy. Dev Med Child Neurol 2005;47:86-93.

9. Jang DH, Choi KH, Kim DH, Lim CM, Kim JS. Comparison between the radionuclide salivagram and videofluoroscopic swallowing study methods for evaluating patients with aspiration pneumonia. Ann Nucl Med 2013;27:247-52.

10. Kim BR, Sung IY, Choi KH, Kim LS, Ryu JS. Long-term outcomes in children with swallowing dysfunction. Dev Neurorehabil 2014;17:298-305.

11. Guidelines for prevention of nosocomial pneumonia.
Centers for Disease Control and Prevention. MMWR Recomm Rep 1997;46(RR-1):1-79.

12. Kawai S, Yokota T, Onozawa Y, Hamauchi S, Fukutomi A, Ogawa $\mathrm{H}$, et al. Risk factors for aspiration pneumonia after definitive chemoradiotherapy or bio-radiotherapy for locally advanced head and neck cancer: a monocentric case control study. BMC Cancer 2017;17: 59.

13. Jo H, Park JG, Min D, Park HW, Kang EK, Lee KJ, et al. Incidence of pneumonia after videofluoroscopic swallowing study and associated factors. Dysphagia 2016; 31:41-8.

14. Smith Hammond C. Cough and aspiration of food and liquids due to oral pharyngeal dysphagia. Lung 2008;186 Suppl 1:S35-40.

15. Henderson M, Miles A, Holgate V, Peryman S, Allen J. Application and verification of quantitative objective videofluoroscopic swallowing measures in a pediatric population with dysphagia. J Pediatr 2016;178:200-5.

16. Baikie G, Reddihough DS, South M, Cook DJ. The salivagram in severe cerebral palsy and able-bodied adults. J Paediatr Child Health 2009;45:342-5.

17. Cook SP, Lawless S, Mandell GA, Reilly JS. The use of the salivagram in the evaluation of severe and chronic aspiration. Int J Pediatr Otorhinolaryngol 1997;41:35361. 